\title{
Proximity Operations and Docking Sensor Development
}

\author{
Richard T. Howard, Thomas C. Bryan and Linda L. Brewster \\ Automated Rendezvous and Docking Development and Test Branch (ES62) \\ NASA, Marshall Space Flight Center, Huntsville, Alabama, 35812 \\ 256-544-3536 ricky.howard@nasa.gov \\ 256-544-3550 tom.bryan@nasa.gov \\ 256-544-0169 linda.brewster@nasa.gov \\ James E. Lee \\ Explorations Advanced Capabilities Office (VP33) \\ NASA, Marshall Space Flight Center, Huntsville, Alabama, 35812 \\ 256-544-4951 james.e.lee@nasa.gov
}

\begin{abstract}
The Next Generation Advanced Video Guidance Sensor (NGAVGS) has been under development for the last three years as a long-range proximity operations and docking sensor for use in an Automated Rendezvous and Docking (AR\&D) system. The first autonomous rendezvous and docking in the history of the U.S. Space Program was successfully accomplished by Orbital Express, using the Advanced Video Guidance Sensor (AVGS) as the primary docking sensor. That flight proved that the United States now has a mature and flight proven sensor technology for supporting Crew Exploration Vehicles (CEV) and Commercial Orbital Transport Systems (COTS) Automated Rendezvous and Docking (AR\&D). NASA video sensors have worked well in the past: the AVGS used on the Demonstration of Autonomous Rendezvous Technology (DART) mission operated successfully in "spot mode" out to $2 \mathrm{~km}$, and the first generation rendezvous and docking sensor, the Video Guidance Sensor (VGS), was developed and successfully flown on Space Shuttle flights in 1997 and 1998. ${ }^{12}$
\end{abstract}

Parts obsolescence issues prevent the construction of more AVGS units, and the next generation sensor was updated to allow it to support the CEV and COTS programs. The flight proven AR\&D sensor has been redesigned to update parts and add additional capabilities for CEV and COTS with the development of the Next Generation AVGS at the Marshall Space Flight Center. The obsolete imager and processor are being replaced with new radiation tolerant parts. In addition, new capabilities include greater sensor range, auto ranging capability, and real-time video output.

This paper presents some sensor hardware trades, use of highly integrated laser components, and addresses the needs of future vehicles that may rendezvous and dock with the International Space Station (ISS) and other Constellation vehicles. It also discusses approaches for upgrading AVGS to address parts obsolescence, and concepts for minimizing the sensor footprint, weight, and

1

${ }^{1}$ U.S. Government work not protected by U.S. copyright

${ }^{2}$ IEEEAC paper \#1287, Version 1, Updated December 17, 2008 power requirements. In addition, the testing of the brassboard and proto-type NGAVGS units will be discussed along with the use of the NGAVGS as a proximity operations and docking sensor.

\section{TABLe of Contents}

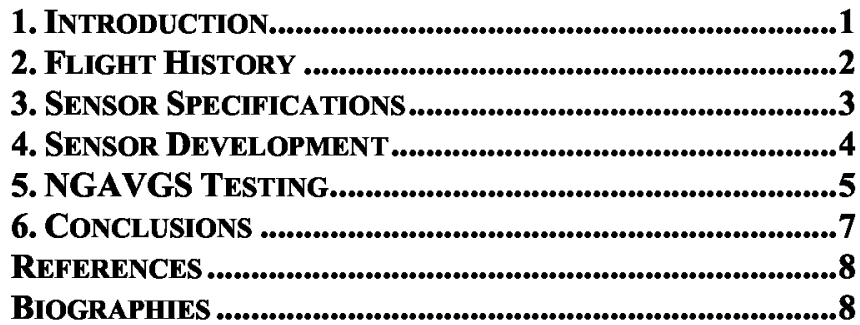

\section{INTRODUCTION}

The NGAVGS is a National Aeronautics and Space Administration (NASA) Marshall Space Flight Center (MSFC) developed sensor capable of supporting automated operations for spacecraft rendezvous, spacecraft proximity operations, spacecraft docking, spacecraft free-flyer capture, fluid transfers, and Orbital Replacement Unit (ORU) transfers. The NGAVGS builds on the technology of the AVGS, incorporating requirements to support the longer ranges required by the Crew Exploration Vehicle (CEV or Orion), up to 5000 meters, and real-time video output. Since one of the key technologies required to support the International Space Station (ISS) re-supply as well as the NASA Constellation Program is Automated Rendezvous and Docking (AR\&D), AR\&D sensor robustness is vital. Thus, radiation is a bigger concern for the current sensor than it was for the Orbital Express (OE) Mission.

As an in-house project, the technologies and testing required for VGS, AVGS and now NGAVGS were developed by engineers within the MSFC Engineering Directorate, continuing the long AR\&D heritage at MSFC. These engineers hold a combined 11 patents on these sensors and related technologies. The NGAVGS flight hardware and software design is also being accomplished by the MSFC Engineering Directorate 
personnel. An internal study was conducted in late 2006 to assess 13 different configuration options for the NGAVGS. The Processor Control Board (PCB) and the imager were key components assessed as a part of this trade study. The recommendation resulting from this trade was a two box configuration with a remote camera head and laser/electronics box that could be internally mounted in the spacecraft to provide radiation protection and thermal dissipation. In addition, a one-box option was also designed to provide an easier to integrate sensor solution. Two different NGAVGS brassboards were built based on the two-box configuration, and two prototypes were built based on the one-box configuration. The brassboard 2/3 is depicted below in Figure 1.

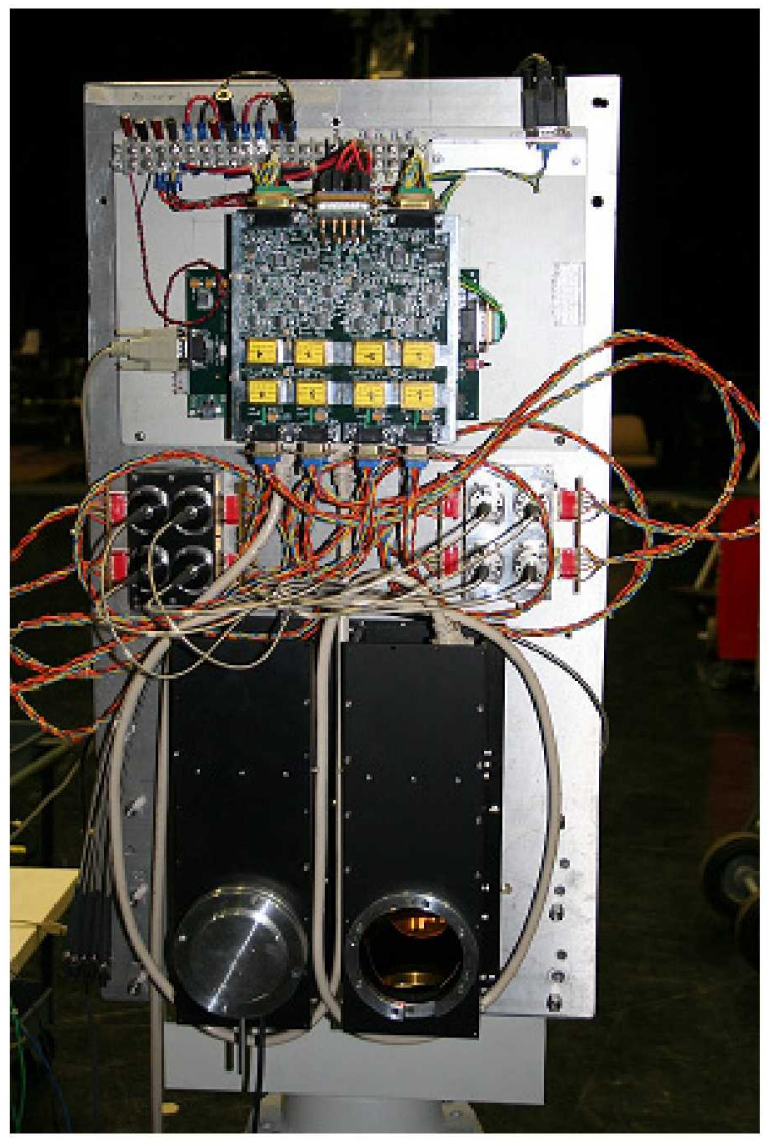

Figure 1: NGAVGS Brassboard 2/3 configuration

\section{FLIGHT HISTORY}

The NGAVGS is the latest sensor in a long line of video sensors developed for automated rendezvous and docking. The proto-type sensors were developed and tested in the MSFC Flight Robotics Laboratory and were initially used to guide a 3-degree-of-freedom (3-DOF) vehicle from an initial position 10 to 20 meters away from the sensor all the way into contact with the target location and throughout the closure of a docking mechanism.
The first sensor built to fly was the Video Guidance Sensor (VGS). It was based on a modified video camera, frame grabber, processor, and software. The VGS was designed as the sensor that would be used to guide a spacecraft the last 100 meters of its AR\&D mission (with the earlier portion of the mission guided by inertial navigation sensors and GPS.) The VGS was flown on the Shuttle on STS-87 and STS-95. It tracked a target that was mounted on a SPARTAN free-flyer. During STS-87, there were some problems with the SPARTAN release, so the VGS only got to track the SPARTAN while it was on the shuttle Remote Manipulator System (RMS). That limited the range to a maximum of about 13 meters. On STS-95, the full mission was carried out, and the VGS got to track the SPARTAN intermittently at 200 meters and constantly from 150 meters in to 10 meters (the minimum approach range). In addition, more data was taken with the SPARTAN mounted on the RMS. [1] [2]

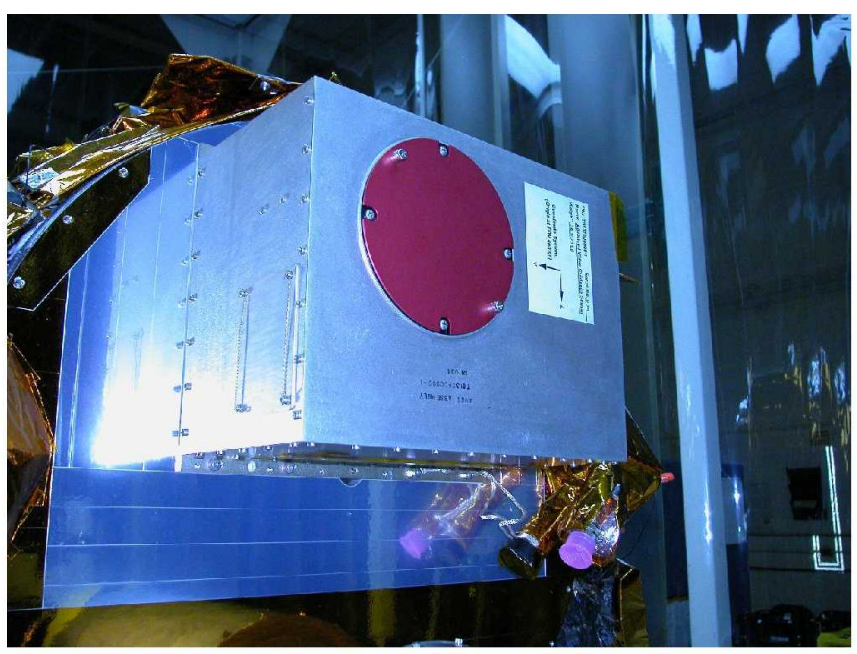

\section{Figure 2: AVGS flight unit mounted on the front of the DART vehicle}

The next generation of sensor was initially developed inhouse, and then the flight version of the hardware was built by Orbital Sciences Corporation. The software was all written, verified, and validated by MSFC's Flight Software group. The Advanced Video Guidance Sensor (AVGS) shown in Figure 2 was 5 times faster than the VGS, used about $1 / 3$ the power, and weighed less than half what the VGS weighed. It was the proximity operations sensor mounted on the front of the Demonstration of Autonomous Rendezvous Technologies (DART) vehicle. The AVGS was capable of acquiring and tracking a target from 300 meters in to docking range (but 4 meters was the closest planned approach range for DART.) While tracking a target, the AVGS provided all six pieces of relative position and attitude information. In addition, the AVGS had a Spot mode in which it would simply provide bearing information to any spots that it saw. This mode worked at ranges up to 2 kilometers 
during the DART mission, but unfortunately the sensor was never commanded into its tracking mode. [3] [4]

An upgraded version of the DART AVGS was desired by Boeing to be used on the Orbital Express (OE) mission. The changes to the sensor were minor, involving tighter thermal controls on some of the optical components and a new mounting location. The software underwent significant improvements, some due to lessons learned on the DART AVGS and some due to new requirements. The DART mission was only supposed to last 24 hours, so there was no need to update the software on orbit. The $\mathrm{OE}$ mission was originally scheduled to be on orbit for up to one year, so the software needed to have the ability to be updated. In addition, there were a number of parameters that may have needed adjusting, so an I-Load (Initial-Load) capability was created so some parameters could be quickly and easily changed without recompiling, validating, and loading a complete set of new software. The OE mission was extremely successful, with the AVGS being used from docking range (about 1 meter) all the way out to 150 meters (despite the fact that the planned use of AVGS was never to have been beyond 120 meters.) [5] [6] [7] [8]

\section{SENSOR SPECIFICATIONS}

The weight, volume, and power consumption estimates for the NGAVGS are on the order of the AVGS values. The AVGS weighed $20 \mathrm{lbs}$, was approximately $7 \times 10 \times$ 12 inches, and consumed approximately 14 Watts in Standby mode and 35 Watts in Tracking Mode (the most power intensive mode of operation). It became clear that to meet the needs of the various mission applications, the MSFC team needed to develop more flexibility into the NGAVGS sensor design packaging. As a result, there is a one box configuration which minimizes the weight and physical integration requirements and a two box design that allows the sensor head to be mounted externally and the laser and electronics box to be mounted internally to reduce environmental effects. The two box concept is shown in Figure 3 below. The NGAVGS one box design is approximately $7 \times 7.5 \times 12$ inches and weighs approximately $8 \mathrm{~kg}$ (17.5 pounds). It consumes an average of less than $30 \mathrm{~W}$ during tracking operations (the most power intense mode of operation). The NGAVGS one box design is shown in Figure 4, and that unit is the second prototype unit built (the Block II Prototype).

The NGAVGS functions in a fashion similar to the AVGS - it takes two sequential pictures illuminated by different wavelengths of laser light, subtracts one picture from the other, creates spots from the lit pixel image data, matches spots to the known target geometry, and computes the relative position vector and relative attitude information.

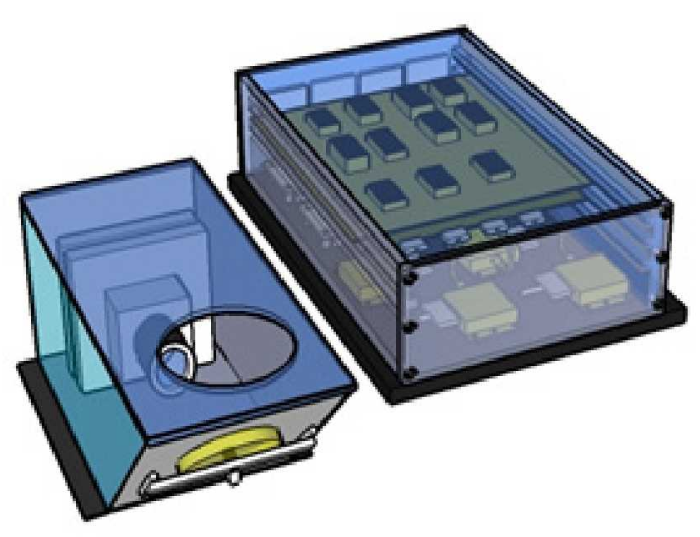

Figure 3: NGAVGS two-box design

During Acquisition or Tracking, image acquisition is initiated by the Digital Signal Processor (DSP). The DSP generates a command to the Field Programmable Gate Array (FPGA) to begin a cycle. The FPGA starts by issuing a fire laser command to the Laser Housekeeping Processor. The lasers are fired and the imager accumulates charge for a predetermined amount of time (the integration time or exposure time). Once the integration time is complete, the image is passed from the imager to the FPGA where it is stored in external memory. Two complete images are accumulated in this manner with each image being stored in separate memory. The differences in the two images are primarily caused by the different reflections of the two different wavelengths of lasers that were fired during the integration time. The two images are then compared by the FPGA, and the lit comparison data is compressed and passed to the DSP where pattern matching is performed. A final solution is calculated and transmitted out thru the RS-422 serial interface. The image can be seen in real time thru the Video Output port. A general block-diagram of the NGAVGS is shown below in Figure 5.

A breadboard version of the NGAVGS was built at MSFC to test the new components that were chosen to replace the obsolete components. Once the breadboard had been successfully tested, two brassboard versions of the NGAVGS were built.

Part of the challenge of upgrading a well performing sensor is confirming any performance changes to the new sensor due to parts that have different performance or configuration. With a new imager and lens and a change in the laser illumination system, the resolution and exposure parameters of the new sensor need to be verified with either old or new target configurations. For the NGAVGS, the Orbital Express Short Range Target and Long Range Target filtered reflectors (OE SRT \& LRT) were selected for the 1 to $200 \mathrm{~m}$ docking range and two International Space Station (ISS) -like hemi-spherical un- 
filtered reflectors for berthing approaches from $5000 \mathrm{~m}$ into $4 \mathrm{~m}$.

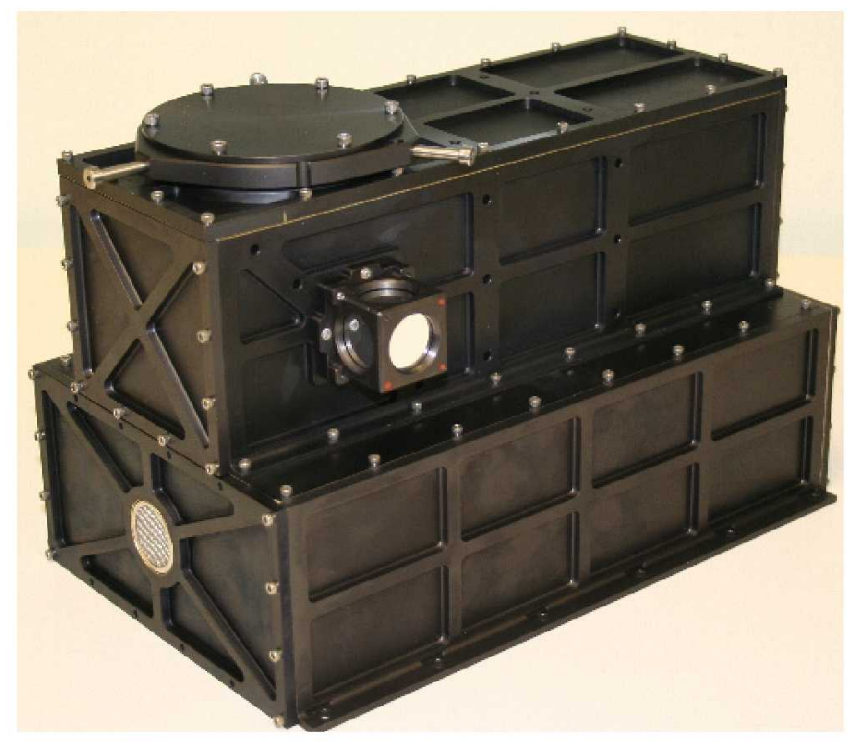

Figure 4: NGAVGS Block II Prototype

\section{SENSOR DEVELOPMENT}

Building on the AVGS laser illumination and imaging technology, the NGAVGS consists of two sets of laser diodes which operate at nominal wavelengths of 806 and $845 \mathrm{~nm}$, a mirror through which the lasers fire, a camera that images the return from the lasers, and hardware, software, and firmware that process the returned images into relative position and attitude data. The sensor is designed to interact with a retro-reflective target. The target has filters that allow one wavelength of AVGS laser to pass through, while blocking the other wavelength. That arrangement causes reflections from the target to occur when one set of lasers is illuminating it, but not when the other set is. The target retro-reflectors are arranged in a pattern known to the AVGS software. The sensor fires the lasers that are passed by the filters and captures an image, and then it fires the second set of lasers and captures a second image. When this second image is subtracted from the first image and an intensity threshold is used, virtually all of the background clutter is eliminated. The remaining lit pixel data is converted into a set of spots, and the spots are compared to the target pattern. Once a set of spots matching the target is found, the software computes the relative position and attitude between the target and the sensor. This data is output from the sensor and fed to the spacecraft Guidance and Navigation System. Figure 5 shows a picture taken down the 300 meter tunnel with the lights on and the lasers in the background mode. Figure 6 shows the same lights on but with the lasers in the foreground mode. Figure 7 is the image in Figure 5 subtracted from the image in Figure
6, showing how the extraneous light, with the two-spot target clearly visible.

As an in-house project, the NGAVGS hardware and software design is being accomplished by the MSFC Engineering Directorate personnel. An initial study was conducted in late 2006 to assess 13 different configuration options for the NGAVGS. The recommendation resulting from this trade was a two box configuration with a remote camera head that would be relatively inexpensive and would be mounted outside the spacecraft and a laser/electronics box that could be internally mounted in the spacecraft to provide radiation protection and thermal dissipation. An NGAVGS brassboard was built based on this configuration, with two different imaging heads to allow for side-by-side performance comparison, and it is depicted in Figure 1.



FIGURE 5: Background Image

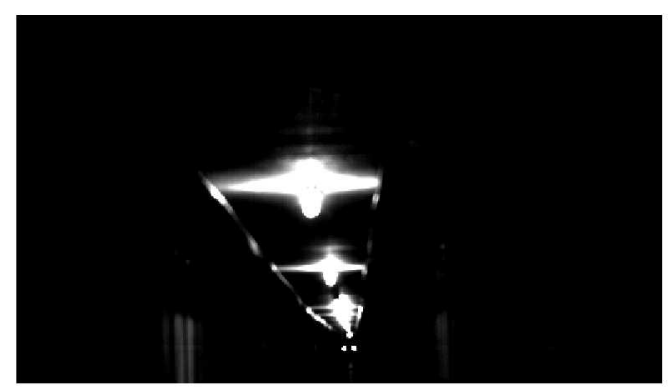

FIGURE 6: Foreground Image with spots At 300 meters

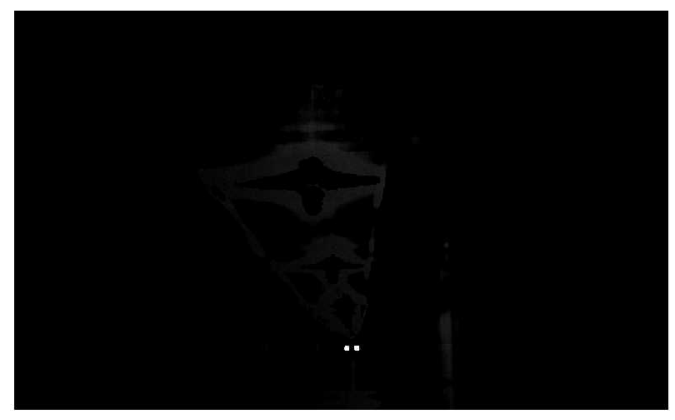

FIGURE 7: Subtracted Image of Spots at $300 \mathrm{~m}$ 
There are several modes of operation for the NGAVGS. The primary NGAVGS modes of operation, based on the OE AVGS modes are as follows: 1) Standby (in which the sensor sends out status messages while awaiting further commands), 2) Acquisition (in which the sensor is commanded to actively seek a target and go into Tracking once a valid target is found), and 3) Tracking (the sensor is actively tracking a target that was found during Acquisition). More information about AVGS can be found in other publications (Howard, 2004).

An initial application for the AR\&D sensor technology for the space station will be to support the COTS for resupply to ISS utilizing ISS/JEM and Node 2 hemispherical targets. The NGAVGS will be able to guide a spacecraft into range to support berthing applications with ISS using the existing ISS Long Range Targets (LRT). As the ISS does not currently have Short Range Targets (SRTs), SRTs would need to be located on the ISS prior to docking applications. An SRT consists of a pattern of retro-reflectors designed to be used from docking range (around 1 meter) out to 15 to 30 meters (depending on the GN\&C system requirements). The SRT shown in Figure 8 is the same size as that used on the OE mission. The AVGS could track that SRT and take relative position and attitude measurements from 1 meter out to 30 meters (demonstrated on orbit as well as in the laboratory.) MSFC has performed numerous target developments and in a new application, a target layout would need to be configured in coordination with the target geometry that would be programmed into the NGAVGS software/firmware. Testing would need to be completed prior to integration with ISS (or any other target spacecraft). ISS hemispherical retro-reflector and an SRT are both shown below in Figure 8 and are utilized in the NGAVGS Optical Characterization Tests (OCTs). Target development is key to the successful use of a sensor for AR\&D. For each Constellation Design Reference Mission (DRM), target layout needs to be assessed and tested because it is integral to the sensor performance.



Figure 8: ISS Hemispherical target (top) with AVGS Short Range Target (bottom)

\section{NGAVGS TESTING}

The first brassboard was used for testing from ranges of 1 meter to 300 meters (the length of the test tunnel). The initial testing consisted primarily of imaging different retro-reflective targets at various ranges and angles to determine the probable overall performance of the initial NGAVGS. The results were that the sensor could see the two separate retro-reflectors at up to 300 meters with a wide variety of integration times and target tilt angles.

The close-range testing with the OE SRT occurred in the Flight Robotics Laboratory, which has a precise computer controlled a two axis sensor gimbal for sensor azimuth and elevation motion and a three axis target gimbal providing target pitch, yaw, and roll positioning. The repeatability and convenience of this testing capability is used for initial focus, alignment, resolution, and exposure parameter testing for various target angles in the center, edge, and corner of the sensor field of view from $1 \mathrm{~m}$ out to $100 \mathrm{~m}$.

The middle range testing occurred in the 300 meter tunnel facility which has undergone renovations and upgrades of 
its own to turn it into a closed-loop computerized test capability. The Apollo-Saturn-era $300 \mathrm{~m}$ underground cable tunnel was cleared of $11,000 \mathrm{ft}$ of cable trays and 200,000 to $300,000 \mathrm{ft}$ of multi-conductor instrumentation cable. A four axis Remote Automated Target Transport (RATT) was designed and built. The RATT positions target mockups with desired yaw, pitch, and roll angles and at positions from 5 to $300 \mathrm{~m}$ in front of the sensor. The sensor can be mounted on a two axis gimbal for testing any portion of the sensor's field-of-view. This facility is controlled by a computer that can move all six axes (range, azimuth, elevation, yaw, pitch, and roll) from static position or rate commands, multi-axis multi-step automated scripts, and simple sensor driven closed-loop dynamic testing. The middle range testing was performed with various retro-reflectors - an $\mathrm{OE}$ type SRT, 1.5 inch diameter OE corner-cube retro-reflectors, and a pair of ISS- like hemispherical arrays of seven 1inch diameter retro-reflectors spaced apart similar to the pair on the bottom of the Japanese pressurized ISS laboratory module, as seen on the top in Figure 8. Figure 9 below shows all of the different targets mounted on the RATT.



Figure 9: ISS-like hemispherical retro-reflectors, OE SRT (bottom left), and 1.5 inch diameter single retroreflector (bottom right)

The second brassboard was used for testing at very long ranges (from 300 meters to 3000 meters). Because there were no available indoor test ranges with the distances required, this testing had to be performed outdoors. Prior to performing the outdoor testing, permission had to be acquired from the Army Redstone Arsenal and from the Federal Aviation Administration (FAA). The very long range testing used Apollo-Saturn-era test stands and towers and the surrounding roadways. This very long range testing was performed with the new sensor and an AVGS EDU shooting out of the eleventh floor window at the top of a test tower and an ISS- like hemispherical array of seven 1-inch diameter retro-reflectors mounted on the elevated handrail of a Saturn 5 engine test stand about $2000 \mathrm{~m}$ south of the test tower and another hemi reflector array mounted on the elevated handrail of a Saturn 1 booster test stand about $3000 \mathrm{~m}$ south of the test tower shown in Fig 10.

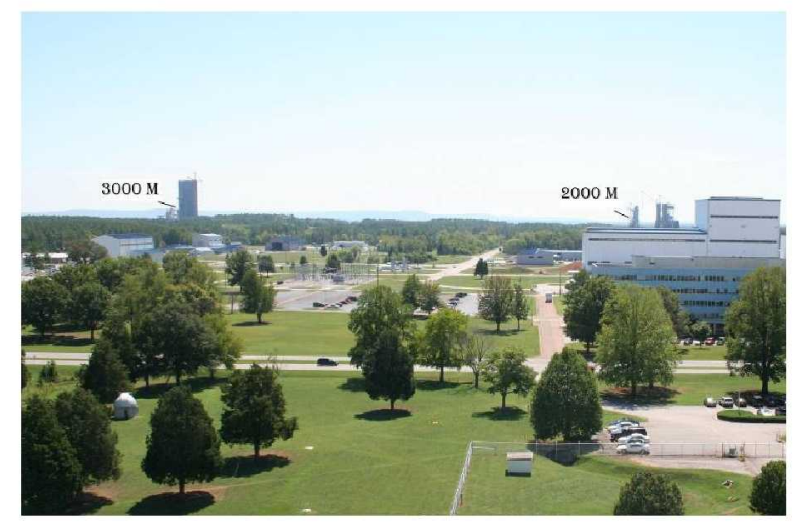

Figure 10: View of $2 \mathrm{~km}$ and $3 \mathrm{~km}$ target stands from test-tower window

There was some definite atmospheric interference that caused the spots to vary in intensity and size for both the new sensor and the baseline sensor - the AVGS Engineering Development Unit (EDU). Spot varied from nothing being visible to a good, bright spot of about 15 pixels. The humidity was greater than $80 \%$, and the high humidity and the dense air caused the atmosphere to interfere with the laser output and the return signal. At $2000 \mathrm{~m}$, the new sensor saw the reflector array with just two lasers at about the same integration time as the AVGS EDU. However, to see the $3000 \mathrm{~m}$ reflector, the new sensor had to have the maximum integration time, which was several times longer than for $2000 \mathrm{~m}$, to clearly see the spot in the center or the edge of the field of view, possibly due to atmospheric conditions or unknown sensor behavior. Successful integration times for 2000 meters were from $8.8 \mathrm{~ms}$ and longer, while at 3000 meters, the sensor only gat spot images at integration times of $65 \mathrm{~ms}$.

Based on the initial brassboard tests, lens and laser changes were evaluated in the brassboard and an engineering unit was designed to meet specifications that were based on the Advanced Video Guidance Sensor performance specifications. A brassboard was first built with the updates to the lasers and lens as well as a higher resolution imager, and it was tested at ranges of 3000 , 
4000 , and 5000 meters. Prior to retesting the unit at $3000+$ meters, a second hemi reflector array was mounted, to allow range measurements also.

At 3000 meters, the atmospheric conditions were very good, and the sensor could track the target quite well. Both the Star-250 and the Star-1000 imagers were tested, and they measured (on the average) ranges within 50 meters of the true range. The standard deviation of the lower-resolution imager was 75 meters, and the standard deviation for the higher-resolution imager was 33 meters. The bearing data was extremely stable, with standard deviations of only 0.002 degrees in azimuth and elevation.

Images taken from the sensor during testing at with targets at $4 \mathrm{~km}$ is shown in Figure 11. Despite the number of street lights and the occasional car, the target spots were clearly visible after the background was subtracted out. The results of the testing were positive. The sensor could detect two separate retro-reflectors at each range, it could give a range and bearing reading, and there was laser power and exposure time to spare. The laser power was set to maximum, but the sensor could see the retro-reflectors even when only one laser was actually used. That means that there is margin in the optical power of the sensor, and it means that the sensor could probably operate at even longer ranges were that a requirement.
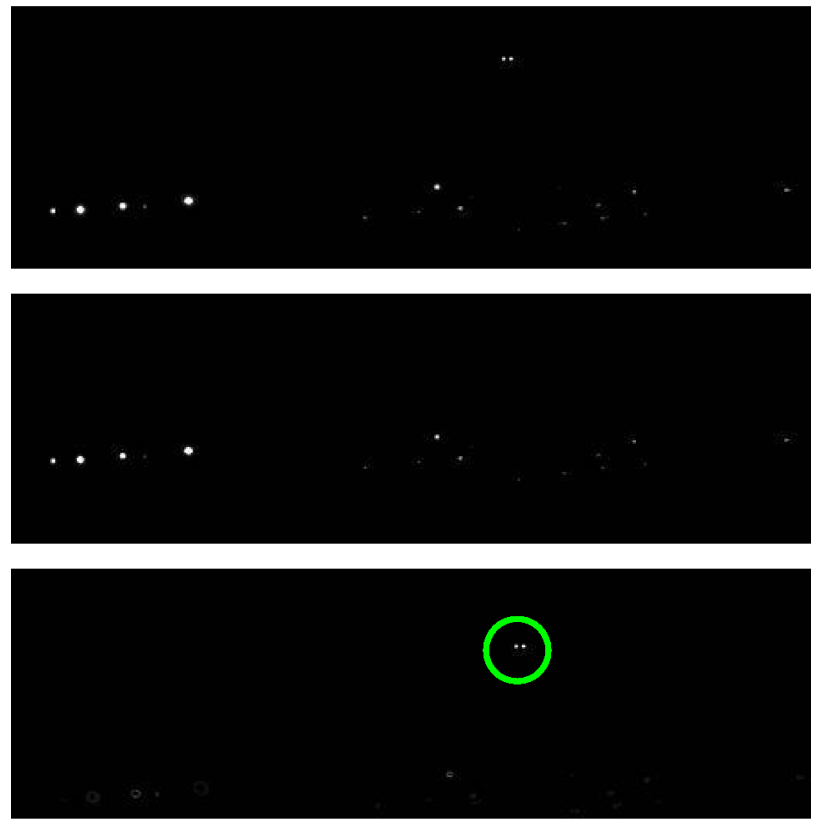

Figure 11: Foreground image (top), background image (middle), and subtracted image with target spots circled (bottom).

A picture of the target (on Hatton Mountain) at $5 \mathrm{~km}$ is shown in Figure 12. The testing was mostly successful. There was a great deal of atmospheric disturbance, causing wide variations in the return spots, but the sensor could image two individual spots and did intermittently track the target. Range values varied $+/-250$ meters (with a static range of exactly 5000 meters) due to the atmospherically-caused noise.

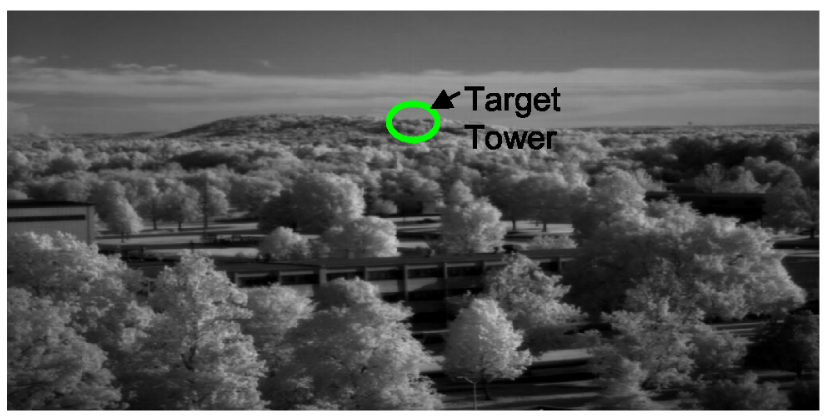

Figure 12: Image taken from NGAVGS of $5 \mathrm{~km}$ target area during daytime. Target tower is barely visible.

During the course of the long range testing, an engineering unit was built. The engineering unit was the Block I prototype, and it was tested in the laboratory as well as in the long range test facility at $3 \mathrm{~km}$. It also functioned well, demonstrating that it could see retroreflectors at that long range, see two distinct retroreflectors, and actually provide range and bearing data to a pair of retro-reflectors spaced the same distance apart as some of the retro-reflectors on the ISS.

The baseline NGAVGS performance requirements are similar to the OE AVGS requirements with regard to range of operation and accuracy $(+/-13 \mathrm{~mm}$ range, $+/-$ 0.033 degrees bearing, $+/-0.3$ degrees attitude), but it must be noted that the sensor performance depends heavily on the target configuration used. The NGAVGS, like its predecessors, looks at the spots of light generated by illuminating a retro-reflective target. The accuracy at which the sensor tracks those spots is a function of reflector size, range, and position in the Field of View.

\section{CONCLUSIONS}

The work performed so far in the design, development, and test of the NGAVGS has been quite successful. The hardware trades resulted in a promising design and the performance tests have shown that the design was a good one. The NGAVGS benefits from the experiences gained from the successful OE AVGS and it is following in the footsteps of its successful progenitors, the DART AVGS and the VGS. The NGAVGS, with the ability to measure bearing out to $5 \mathrm{~km}$ and measure relative 6-DOF information at closer ranges, is able to support the Constellation and COTS programs. 


\section{REFERENCES}

[1] Howard, R. T., Bryan, T. C., and Book, M. L., "The Video Guidance Sensor - A Flight Proven Technology," in Proceedings of the $22^{\text {nd }}$ Annual American Astronautical Society Guidance and Control Conference, 1999.

[2] Howard, R. T., Bryan, T. C., and Book, M. L., "On-Orbit Testing of the Video Guidance Sensor," in Proceedingsof SPIE Laser Radar Technology and Applications IV, 1999.

[3] Howard, R. T., and Bryan, T. C., "DART AVGS Flight Results," in Sensors and Systems for Space Applications, edited by R. T. Howard and R. D. Richards, SPIE Conference Proceedings Vol. 6555, Bellingham, WA, 2007, pp. 1-10.

[4] Howard, R. T., Johnston, A. S., Bryan, T. C., and Book, M. L., "Advanced Video Guidance Sensor (AVGS) development testing," in Spaceborne Sensors, edited by R. D. Habbit, Jr. and P. Tchoryk, Jr., SPIE Conference Proceedings Vol. 5418, Bellingham, WA, 2004, pp. 50-60.

[5] Kennedy, F. G. III, "Orbital Express: Accomplishments and Lessons Learned," in proceedings of 31st Annual AAS Guidance and Control Conferenece, 2008, paper AAS 08-071.

[6] LeCroy, J. E., Hallmark, D. S., and Howard, R. T., "Effects of Optical Artifacts in a Laser-Based Spacecraft Navigation Sensor," in Sensors and Systems for Space Applications, edited by R. T. Howard and R. D. Richards, SPIE Conference Proceedings Vol. 6555, Bellingham, WA, 2007, pp. 1-11.

[7] LeCroy, J. E., Hallmark, D. S., Scott, P., and Howard, R. T., "Comparison of navigation solutions for autonomous spacecraft from multiple sensor systems," in Sensors and Systems for Space Applications II, edited by R. T. Howard and P. Motaghedi, SPIE Conference Proceedings Vol. 6958, Bellingham, WA, 2008, paper [6958-13].

[8] Mulder, T., "Orbital Express Autonomous Rendezvous and Capture Flight Operations," AAS/AIAA Space Flight Mechanics Meeting, Galveston, TX, Jan. 27-31, 2008. Paper AAS 08-209.

\section{BIOGRAPHIES}

Richard T. (Ricky) Howard is a senior engineer at

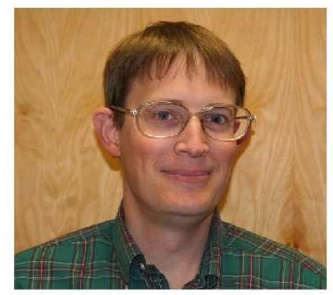
NASA's Marshall Space Flight Center. He is the team leader for the Advanced Vehicle Sensors Team in the Automated Rendezvous and Docking Branch. He has worked on several video-based sensors that have been flown on different spacecraft. He has earned 13 patents, authored or coauthored many conference papers, and been the Contracting Officer's Technical Representative on several SBIRs. He earned a BS in Electrical and Computer Engineering in 1986 and an MS in Control Theory in 1991, both at the University of Alabama in Huntsville.
Thomas C. Bryan is a Senior Engineer at Marshall Space Flight Center's Flight Robotics Laboratory with over thirty years providing engineering support and technology development to numerous orbital docking and

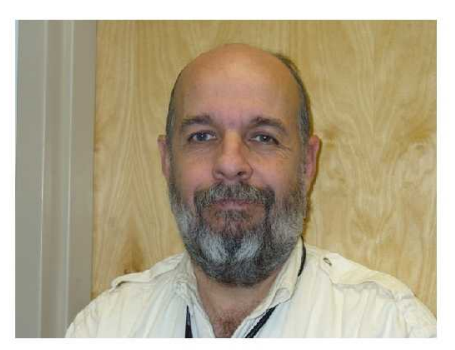
servicing programs. CoInventor of Video Guidance Sensor (VGS) and VGS Targets, Principal Investigator for two Shuttle Flight Experiments and for the Demonstration of Autonomous Rendezvous Technology (DART) Advanced Video Guidance Sensor (AVGS). He is the technical lead on development and testing for the NGAVGS. He is a holder of 10 AR\&D sensor and target patents and an Agency expert on Rendezvous and Docking sensors (foreign and domestic) and GN\&C implementation and testing. He has a B.S. in Electrical Engineering from the University of Tennessee, Knoxville.

James E. Lee is the Project Manager of the Automated Rendezvous and Docking Projects Team in the Science and Mission Systems Directorate at the NASA Marshal

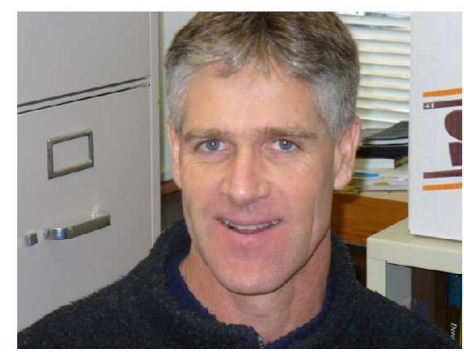
Space Flight Center. He leads a team of civil service and support contractor employees whose AR\&D Mission is the development and testing of critical AR\&D components and systems to enable future assisted or automated spacecraft rendezvous and docking maneuvers. Previously he served as project manager for the NASA Orbital Express Advanced Video Guidance Sensor (AVGS) Project and the Hypersonic Technology Experiment Project. $\mathrm{He}$ is a graduate of Auburn University with a B.S. in Chemistry and a graduate of the University of Alabama in Birmingham with a B.S. and Masters in Materials Engineering.

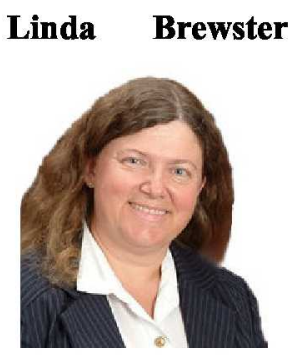
is the

Autonomous Applications Simulation Lab which supported SEDS, TSS-1 and TSS-1R missions. She has a BSEE from New Mexico State University and a MSEM from Florida Institute of Technology. 
